\title{
Index of deterioration of patients with mechanical prosthetic heart valve thrombosis
}

\author{
Ahmed Hassouna, Mohamed El-Ghanam * (D, Hasan Moftah, Khaled Samir and Khaled Refaat
}

\begin{abstract}
Background: Mechanical prosthetic heart valves are known for their durability; however, a malfunctioning prosthesis can deteriorate rapidly to become a life-threatening complication. Our aim was to calculate a numerical index to express the rate of clinical deterioration of patients presenting with a mechanical prosthetic heart valve thrombosis (PVT), called the index of deterioration (ID), and to evaluate its usefulness in predicting hospital outcomes.

Results: The median ID and range were $(0.43,0.03-3)$ NYHA class/day. A higher ID was significantly related to early development of PVT after native valve replacement, younger age, female gender, pregnancy, non-compliance to oral anticoagulation (OAC), low LVEF\%, high mean pressure gradient across a mitral prosthesis, raised serum creatinine, and SGOT on admission $(P<0.05)$. Independent predictors were early presentation after native valve replacement, female gender, and non-compliance to OAC $(P<0.05)$.

ID correlated positively with the need for urgent/emergency surgery, and the durations of cardiopulmonary bypass, postoperative mechanical ventilation, and positive inotropes. ID correlated negatively with the postoperative LVEF\% $(P<0.05)$. Median ID of the 21 mortalities $(0.75,0.1-3)$ was $>2.1$ times that of survivors $\left(0.35: 0.03^{-2} ; P=0.002\right)$, and the median ID of the 29 cases with postoperative complications $(0.5,0.1-1.5)$ was 2.5 times that of the 39 uneventful cases $(0.2,0.03-2 ; P=0.011)$. The ID significantly predicted both mortality (odds ratio $3.87 ; 1.33-1.29 ; P=0.013$ ) and mortality and hospital complications (odds ratio 4.77; 1.49-15.2; $P=0.008$ ). The respective discriminating abilities were AUC 0.734 $(0.616-0.852 ; P=0.002)$ and $0.724(0.61-0.835 ; P<0.001)$. EuroScore II correlated positively with ID $(r=0.571 ; P<0.001)$ but showed better discriminative abilities.
\end{abstract}

Conclusion: The simple index of deterioration was useful in monitoring deterioration and predicting hospital progression and outcomes in patients presenting with PVT.

Keywords: Prosthetic valve malfunction, Prosthetic valve thrombosis, Mechanical valve thrombosis, Scoring systems, EuroScore II

\section{Background}

In our experience, mitral valve repair is not always feasible in the young rheumatic population and valve replacement with a mechanical valve is preferred over the use of a bioprosthesis, which is likely to degenerate over time and require reintervention [1]. Although mechanical valves have long term durability, yet the incidence of malfunction is not negligible $(0.1-6 \%)$ and mechanical prosthetic valve thrombosis (PVT) is one of those serious complications, where patients often deteriorate rapidly, compromising management outcomes. The aim of this study was to express the rate by which those patients deteriorate clinically by a simple numerical index and to test its value in monitoring hospital progression and predicting outcomes of surgery.

\footnotetext{
* Correspondence: mohamedelghanam@yahoo.com

Cardiothoracic Surgery Department, AinShams University, Cairo, Egypt
}

\section{Springer Open}

(c) The Author(s). 2020 Open Access This article is licensed under a Creative Commons Attribution 4.0 International License, which permits use, sharing, adaptation, distribution and reproduction in any medium or format, as long as you give appropriate credit to the original author(s) and the source, provide a link to the Creative Commons licence, and indicate if changes were made. The images or other third party material in this article are included in the article's Creative Commons licence, unless indicated otherwise in a credit line to the material. If material is not included in the article's Creative Commons licence and your intended use is not permitted by statutory regulation or exceeds the permitted use, you will need to obtain permission directly from the copyright holder. To view a copy of this licence, visit http://creativecommons.org/licenses/by/4.0/. 


\section{Methods}

This prospective study included 86 patients with PVT who were operated upon in our hospital between January 2016 and October 2019. Written informed consent was obtained from all participants in this study. Inclusion criteria were patients of both sexes presenting with symptoms and or signs suggesting PVT that was documented by transthoracic as well as transesophageal echocardiography on hospital admission. We have excluded cases with recurrent PVT as well as the subset of patients who presented within a short period (1-2 weeks) of the development of mild symptoms (NYHA class I or II), had a small thrombus on echocardiography $(<1 \mathrm{~cm})$ and who responded positively after a short period (1-2 days) of continuous IV heparinotherapy or fibrinolytic therapy.

History intake included date of native valve replacement type and size of implanted prosthesis and any history of valve or anticoagulant related complications. We suggested that every prosthesis is doomed to become malfunctioning 1 day or another and hence, the period that elapsed between the date of implantation of the prosthesis and the appearance of the signs and or symptoms suggesting PVT was recorded in months and was called the latent period of malfunction (LPM). The usually much shorter period that follows afterward and till the patient is reoperated was recorded in days and it was called the manifest period of malfunction (MPM). The patient's medications were reviewed, especially his compliance to oral anticoagulation therapy (OAC). Noncompliance was defined as a patient skipping $>2$ successive doses, changing dose, stopping intake, or shifting to another anticoagulation regimen, without cardiological consultation. In order to calculate the index of deterioration (ID), we assigned the numbers $1,2,3$, and 4 to the patient's NYHA class I, II, III, and IV respectively. The change in NYHA class for a particular patient (delta NYHA) was calculated by subtracting the baseline numerical NYHA class value before the event form the numerical NYHA class value of the patient at presentation. The patient's ID was then calculated by dividing the delta NYHA by the duration of the MPM in days.

Once admitted, all patients benefited from routine preoperative preparation. Female patients were assessed for their pregnancy state and pregnant cases received immediate obstetric evaluation. Based on the clinical and echocardiographic findings, the timing of surgery was decided by the attending surgeon. Surgery was performed through median sternotomy under cardiopulmonary bypass, moderate hypothermia, and antegrade cold blood cardioplegia. The prosthesis was inspected, with the primary intention to be replaced by a mechanical prosthesis in most of the cases. The use of a bioprosthesis or limited thrombectomy was left to the personal surgeon's judgment and patient preference. Operative survivors were transported to the ICU and managed routinely till discharge.

\section{Statistical analysis}

Qualitative variables were presented as numbers and percentages, and the significance of their association was tested by chi-square or Fisher's exact test, as indicated. Shapiro-Wilk test showed a significant deviation of normality and calculated ID scores were hence expressed as medians and range. The significance of their association was tested by Spearman's correlation rank test and groups were compared by Mann and Whitney test or Kruskal and Wallace test, as indicated.

To evaluate the discriminative ability of ID, we have calculated EuroScore II for our patients and both scores were subjected to the receiver-operating characteristic analyses, calculating the area under the curve (AUC) for hospital mortality as well as both hospital mortality and complications. In addition, univariate logistic regression analyses were run to evaluate the predicted probability of ID and EuroScore or hospital mortality as well as model calibration by the Hosmer-Lemeshow test for the lack of "goodness of fit."

In order to detect the independent preoperative predictors of clinical deterioration (ID), variables that were significantly related to ID on univariate analysis were introduced in a multiple linear regression model. The dependent variable was the normalized values of ID, calculated by the 2 steps approach described by Templeton GF, which involves ranking cases followed by normalization of ranks with the inverse distribution function using fractional rank, mean, and standard deviation [2]. Normality was then rechecked by the Shapiro-Wilk test. All tests were bilateral, and a $P$ value of $5 \%$ was the limit of statistical significance. The analysis was performed by the statistical package software IBM-SPSS version 25 (IBM Corp, Chicago, IL, USA).

\section{Results}

Table 1 shows the patients demographics. The median age was 49 (16-74) years, with a female predominance and a majority of patients having a single mechanical bileaflet prosthesis implanted in the mitral position (67 cases, $78 \%$ ). The main presenting symptom was dyspnea and palpitation and $96.5 \%$ of cases presented with NYHA class III or IV. Two-thirds of patients were not compliant to OAC regimen on admission, including all the 16 pregnant cases included in our study. Eight of those cases $(75 \%)$ reduced their recommended warfarin dose or completely stopped the intake for fear of warfarin-related embryopathy. The other 4 cases were shifted to inadequate heparin therapy by their attending obstetrician, without cardiological consultation. There 
Table 1 Patients' demographics $(n=86)$

\begin{tabular}{|c|c|}
\hline Variable & \\
\hline Age (years) & $40(16-74)$ \\
\hline Female gender & $52(60.5 \%)$ \\
\hline Pregnancy & $16(18.6 \%)$ \\
\hline Number of implanted prostheses & 91 \\
\hline Mechanical bileaflet & 85 (93.4\%) \\
\hline Mechanical mono-leaflet & $4(4.4 \%)$ \\
\hline Additional tricuspid bioprosthesis* & $2(2.2 \%)$ \\
\hline \multicolumn{2}{|l|}{ Position of implantation } \\
\hline Mitral & $68(79.1 \%)$ \\
\hline Aortic & $13(15.1 \%)$ \\
\hline Mitral and aortic & $3(3.5 \%)$ \\
\hline Mitral + tricuspid bioprosthesis* & $2(2.3 \%)$ \\
\hline Non-compliance to OAC & $53(61.6 \%)$ \\
\hline \multicolumn{2}{|l|}{ Main presenting symptom: } \\
\hline Dyspnea and palpitation & $70(81.4 \%)$ \\
\hline Thromboembolic event & $12(14 \%)$ \\
\hline Threatened abortion & $4(4.6 \%)$ \\
\hline \multicolumn{2}{|l|}{ NYHA class at presentation } \\
\hline Class IV & $47(54.7 \%)$ \\
\hline Class III & $36(41.9 \%)$ \\
\hline Class ॥ & $3(3.5 \%)$ \\
\hline Numerical NYHA class & $3.5(2-4)$ \\
\hline LVEF\% & $57.5(22-73)$ \\
\hline $\begin{array}{l}\text { Mean pressure gradient across mitral prosthesis ( } \mathrm{mm} \\
\mathrm{Hg} \text { ) }\end{array}$ & $12(9-20)$ \\
\hline $\begin{array}{l}\text { Mean pressure gradient across aortic prosthesis ( } \mathrm{mm} \\
\mathrm{Hg} \text { ) }\end{array}$ & $54(5-80)$ \\
\hline INR on admission & $1.55(0.7-4.2)$ \\
\hline Serum creatinine (mg/dl) & $1.37(0.6-4.2)$ \\
\hline SGOT (units/liter) & $45(15-150)$ \\
\hline Latent period** (months) & $44.6(0.77-365)$ \\
\hline Index of deterioration (ID) & $0.43(0.03-3)$ \\
\hline EuroScore II & $\begin{array}{l}6.75(1.69- \\
26.5)\end{array}$ \\
\hline
\end{tabular}

Values are presented as number (\%) or median and range $n$ number of patients, PVT prosthetic valve thrombosis, LVEF\% left ventricular ejection fraction percentage

*Well-functioning bioprosthesis

**Time between implantation of mechanical prosthesis and development of symptoms and/or signs suggesting PVT

was a statistically significant negative correlation between the index of deterioration and the time elapsed between native valve replacement and LPM (Fig. 1). The earlier the patient developed the malfunction, the faster he deteriorated $(r=-0.489 ; P<0.001)$.

Table 2 shows selected operative and postoperative outcomes. Only 2 patients were operated upon electively after presenting with progressive dyspnea and echocardiographic data showing a small thrombus, 60 and 180 months after mitral valve replacement with a SJ bileaflet mechanical prosthesis. The 2 patients were operated upon after failure to improve from a brief 2-days trial of heparin therapy followed by fibrinolytic therapy. Out of the $91 \mathrm{im}$ planted prostheses, 86 were malfunctioning (94.5\%), 80\% of which were replaced with a bileaflet mechanical prosthesis. The use of a bioprosthesis was limited to the 16 cases $(18.6 \%)$ who either preferred to avoid the life-long OAC regimen, as well as those patients having a history of repeated non-compliance to OAC. On the other hand, management was limited to thrombectomy and careful cleaning of the valve housing in one case presenting with a small recent thrombus and a low INR of 1.4 on admission. Unfortunately, the patient died postoperatively from persistent low cardiac output, with an apparently wellfunctioning prosthesis on transesophageal echocardiography. Our overall hospital mortality was high (21\%) and 29 patients had non-fatal in-hospital complications (33.7\%). A total of 49 complications were recorded in those cases including significant postoperative bleeding in 8 patients $(16.3 \%)$, which necessitated reoperation in 2 cases $(4 \%)$, prolonged mechanical ventilation in 7 cases (14.3\%), need for reintubation in 4 cases $(8.2 \%)$, temporary heart block in 6 cases (12.2\%), low cardiac output in 5 cases $(10.2 \%)$, cerebrovascular stroke in 2 cases (4\%), septic shock in 2 cases (4\%), multiorgan failure in 2 cases (4\%), and acute renal failure necessitating dialysis in another case (2\%). Ten adverse fetal outcomes were recorded among the 16 pregnant patients (20.4\%), included 4 cases of abortion (8.2\%), and 6 fetal losses (12.2\%).

Table 3 shows the ID values for patients' subgroups and their correlation with the patients' demographics, associated risk factors, and hospital outcomes. Younger age on admission, female gender, pregnancy, and noncompliance to $\mathrm{OAC}$ were all significantly associated with a high ID. A high ID significantly correlated with increased mean pressure gradient across a malfunctioning mitral prosthesis, need for urgent or emergency surgery, and prolonged durations of cardiopulmonary bypass, postoperative mechanical ventilation, and inotropic support. A high ID was significantly associated with hospital mortality as well as the development of complications among survivors.

Table 3 also shows the calculated EuroScore II values that nearly followed the same pathway as the ID, with a statistically significant positive correlation between both indices $(r=0.571 ; P<0.001$; Fig. 2). EuroScore II correlated negatively with LPM and MPM and positively with NYHA class at presentation as well as the need for emergency surgery. A high EuroScore II was also significantly related to mortality and to the development of complications among hospital survivors. Unlike the 


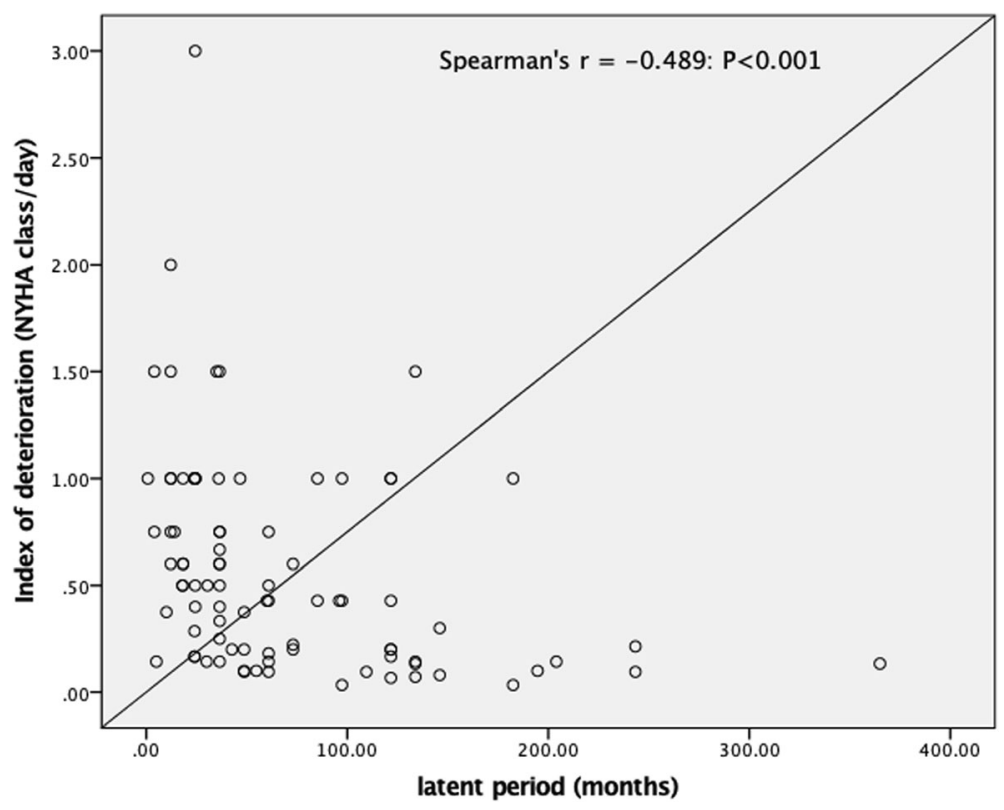

Fig. 1 Correlation between index of deterioration and latent period

index of deterioration, EuroScore II did not significantly correlate with the patient's demographics at presentation, namely, age, gender, pregnancy status, and noncompliance to OAC.

Preoperative variables that were statistically significant on univariate analysis were introduced in a linear regression model to detect the independent preoperative predictors of ID, using the normalized ID as the dependent variable. The mean normalized ID + standard deviation was $0.56 \pm 0.48$, the normalized ID of mortalities $(0.82 \pm$ 0.33 ) was significantly higher than that calculated for survivors $(0.5+0.49 ; P=0.003)$. Out of the nine statistically significant variables shown in Table 3 , only six were introduced in the model, namely, age on admission, gender, pregnancy state, non-compliance to $\mathrm{OAC}$, mean pressure gradient across the prosthesis, and LPM. Both NYHA class and MPM were excluded for the high colinearity due to their use in the direct calculation of the dependent variable itself. The urgency of the operation was not used for being the result rather than being the predictor of the deterioration. The model significantly explained ID, $R^{2}=0.33 ; P<0,001$. The independent predictors of high ID were female gender $(B=0.236$ : 0.042$0.43 ; P=0.018)$, non-compliance to $\mathrm{OAC}(0.215,0.031$ $0.4 ; P=0.023)$, and early presentation of PVT as indicated by a short latent period of malfunction $(-0.003$ : -0.004 to $-0.002 ; P<0.001$ ).

Table 4 shows variables that were significantly related to hospital mortalities in univariate analysis. In order to detect the independent preoperative predictors of mortality variables that were statistically significant on univariate analysis were introduced in a logistic regression model. The model predicted mortality (sensitivity $96.4 \%$, specificity $57.1 \%$, and Negelkerke $R$ square 0.577 ; $P<0.001$ ); data fitted the model (Hosmer-Lemeshow test; $P=0.7)$ and the only independent predictor was low LVEF\% on admission (odds ratio 0.85, 0.75-0.96; $P$ = 0.008 ). The model was not improved by the introduction of either ID or EuroScore or both and none of them significantly predicted mortality.

Although our observed $21 \%$ hospital mortality was nearly triple that expected by EuroScore II (mean, 7.3\%, median, 6.7\%, range, 1.69-26.5, and 95\% interval of confidence of median, 4-84-6.89); $P<0.001$, yet the discriminative ability of the later (AUC 0.799, 0.69-0.9; $P<$ 0.001 ) was better than that calculated with ID (AUC $0.734,0.62-0.75 ; P=0.002$ ), as shown in Fig. 3. Univariate logistic regression analysis showed that both scores significantly predicted mortality but the EuroScore odds ratio 1.35 (1.13-1.61); $P=0.001$ and Hosmer and Lemeshow test; $P=0.345$; demonstrated more accurate predictive ability than those calculated for ID: odds ratio, 3.87 (1.3-11.3); $P=0.013$; and Hosmer and Lemeshow test $P=0.07$. As shown in Fig. 4, better discriminative ability for hospital mortality and complications was also recorded with EuroScore II (AUC 0.799: 0.69-0.9; $P<$ 0.001 ) than with ID (AUC 0.734, 0.62-0.85; $P<0.002$ ). Univariate logistic regression analysis showed that both scores significantly predicted mortality but the EuroScore odds ratio 1.36 (1.15-1.62); $P<0.001$ and Hosmer and Lemeshow test; $P=0.08$; demonstrated more accurate predictive ability than that calculated for ID: 
Table 2 Selected operative and postoperative outcomes

\begin{tabular}{|c|c|}
\hline Variable & \\
\hline \multicolumn{2}{|l|}{ Schedule of surgery } \\
\hline Emergency & $51(59.3 \%)$ \\
\hline Urgent & $33(38.4 \%)$ \\
\hline Elective & $2(2.3 \%)$ \\
\hline \multicolumn{2}{|l|}{ Management of malfunctioning prosthesis* } \\
\hline Replacement with mechanical prosthesis & $69(80.2 \%)$ \\
\hline Replacement with bioprosthesis & $16(18.6 \%)$ \\
\hline Thrombectomy alone & $1(1.2 \%)$ \\
\hline Aortic cross clamp time (min) & $61(37-200)$ \\
\hline Cardiopulmonary bypass time (min) & $\begin{array}{l}110(55- \\
360)\end{array}$ \\
\hline Difficulty to wean from bypass & $25(29.1 \%)$ \\
\hline Duration of mechanical ventilation (h) & $24(6-168)$ \\
\hline Duration of inotropes $(h)$ & $48(0-216)$ \\
\hline Transfused blood products (I) & $4(2-12)$ \\
\hline ICU stay (days) & $4(1-39)$ \\
\hline Total hospital stay (days) & $10(1-40)$ \\
\hline Postoperative LVEF\% & $54(22-70)$ \\
\hline \multicolumn{2}{|c|}{$\begin{array}{l}\text { Postoperative pressure gradient across prosthesis ( } \mathrm{mm} \\
\mathrm{Hg} \text { ) }\end{array}$} \\
\hline Mitral & $5(3-10)$ \\
\hline Aortic & $20(15-50)$ \\
\hline Hospital mortality & $18(21 \%)$ \\
\hline Low cardiac output & $-8(44.4 \%)$ \\
\hline Multi organ failure & $-4(22.2 \%)$ \\
\hline Septic shock & $-6(33.3 \%)$ \\
\hline Uneventful cases & $39(45.3 \%)$ \\
\hline
\end{tabular}

Values are presented as numbers (\%) or median (range) LVEF\% left ventricular ejection fraction percentage *Out of the 91 implanted prostheses, only 86 were malfunctioning (94.5\%)

odds ratio, 3.87 (1.5-15.2); $P=0.008$; and Hosmer and Lemeshow test $P=0.05$.

\section{Discussion}

Every foreign material implanted in the circulation invites thrombosis and hence, PVT is expected to remain an inherent complication of heart valve replacement. Put it another way, every mechanical prosthesis is assumed to develop PVT 1 day or another, which is why we have termed the period extending from the implantation of the prosthesis and till the development of the signs and/ or the symptoms suggesting PVT as the latent period of malfunction (LPM). In a study that included 680 consecutive patients undergoing mechanical mitral valve replacement, routine transesophageal echocardiography performed at day 9 identified thrombus in as much as $9.4 \%$ of patients. Although $97 \%$ of those patients were successfully managed by anticoagulant therapy, yet 2 cases of PVT needed intervention [3].

Pathologically, the formation of a thrombus on the prosthetic structures reduces the effective orifice area and increases the trans-valvular gradient, producing a variety of symptoms starting from progressive shortness of breath and palpitation and up to a fulminating congestive heart failure. In addition, the hemodynamic disturbance involves the creation of areas of turbulence that increases shear stress and endothelial injury, and adjacent areas of stasis that increases blood coagulability (both inviting more thrombosis [4]) and can generate thromboembolic events $[5,6]$.

There are several mechanisms by which PVT occur, varying from a fast process commencing early after implantation, to the generation of vegetation during the process of endocarditis or the formation of thrombi with the slow ingrowth of a fibrotic pannus over the years [5, 7]. Blackstone and Kirklin have shown that prosthetic valve-related adverse outcomes are not only dependent upon the underlying pathology but also on the timing of presentation [8]. In consequence, we expected that the different pace of those mechanisms will be reflected on the pattern of clinical presentation, especially the length of the period that starts with the patient developing symptoms and/or signs suggesting PVT and ends by reoperation: the manifest period of malfunction (MPM). We expected that the number of NYHA classes the patient loses during this period will objectively reflect the ongoing pathology and, hopefully, may be useful in predicting hospital outcomes. We can easily note that the operative risk of a patient who progresses slowly to reach NYHA class IV over a relatively long period of time is in many times different from another NYHA class IV patient whose prosthesis was catastrophically obstructed by a large thrombus. In fact, the importance of both deterioration in NYHA class and the rate by which it occurs was previously noted by Husebey and colleagues who suggested that advanced NYHA functional class and need for emergency (rapid rate of progression) have to be treated separately [9]. Our suggested ID overcomes this separation as it expresses both the size of deterioration (advancement in NYHA class) and its rate (MPM) in a single numerical value.

The annual rate of PVT is estimated to be between 0.1 and $5.7 \%$, with higher rates being observed in the early perioperative period $[10,11]$, with prosthesis implanted in the mitral and tricuspid position [5, 12], in association with subtherapeutic anticoagulation [11-15] or interruption of oral anticoagulants for anticipated non-cardiac surgery [16] or during pregnancy $[15,17]$. Predominance of female gender was reported in 2 large series $[8,11]$. Blackstone and Kirklin identified female gender as the only risk factor that was significantly associated with acute prosthetic thrombosis [8]. 
Table $\mathbf{3}$ Index of deterioration versus EuroScore values

\begin{tabular}{|c|c|c|c|c|}
\hline \multirow[b]{2}{*}{ Variable } & \multicolumn{2}{|l|}{ Index of deterioration (ID) } & \multicolumn{2}{|l|}{ EuroScore II } \\
\hline & Effect size & $P$ value & Effect size & $P$ value \\
\hline \multicolumn{5}{|l|}{ A-Preoperative (on admission) } \\
\hline Age (years) & $r=-0.245$ & 0.023 & $r=0.039$ & 0.73 \\
\hline \multicolumn{5}{|l|}{ Gender } \\
\hline Females versus males & 0.55 (0.07-3) vs. 0.21 (0.03-1.5) & 0.003 & $8.4(2.9-26.5)$ vs. 5 (1.69-15) & 0.13 \\
\hline Non-pregnant females vs. males* & $0.46(0.07-1.5)$ vs. $0.23(0.03-1.5)$ & 0.05 & $8.4(2.9-26.5)$ vs. 5 (1.69-15) & 0.27 \\
\hline Pregnancy: pregnant vs. other cases & $0.87(0.3-3)$ vs. $0.39(0.03-1.5)$ & 0.003 & $8.4(3.4-11.2)$ vs. 6.5 (1.7-26.5) & 0.13 \\
\hline \multicolumn{5}{|l|}{ Position } \\
\hline Mitral + tricuspid & $2(2-2)$ & & $11.5(7.9-15)$ & \\
\hline Mitral + aorta & $1(0.07-1)$ & & $6.6(6.4-10.5)$ & \\
\hline Single mitral & $0.46(0.07-3)$ & & $6.9(2.8-18)$ & \\
\hline Single aortic & $0.3(0.03-1)$ & 0.43 & $6.4(1.7-26.5)$ & 0.47 \\
\hline Non-compliance to OAC: yes vs. no & $0.5(0.1-3)$ vs. $0.33(0.03-1.5)$ & 0.03 & 6.9 (2.8-18) vs. $6.4(1.7-26.5)$ & 0.36 \\
\hline Thromboembolic events: yes vs. no & 0.29 (1-1.5) vs. $0.5(0.03-3)$ & 0.35 & 8.4(2.9-11.4) vs. 6.6 (1.7-26.5) & 0.99 \\
\hline LVEF\% at presentation & $r=-0.155$ & 0.155 & $r=-0.44$ & $<0.001$ \\
\hline \multicolumn{5}{|l|}{ Mean gradient across prosthesis } \\
\hline Mitral & $r=0.52$ & $<0.001$ & $r=0.325$ & 0.007 \\
\hline Aortic $(\mathrm{mm} \mathrm{Hg})^{* *}$ & $r=-0.44$ & 0.129 & $r=-0.53$ & 0.061 \\
\hline \multicolumn{5}{|l|}{ NYHA functional class } \\
\hline Class II & $0.1(0.03-0.14)$ & & $2.86(1.69-3.7)$ & \\
\hline Class III & $0.17(0.03-2)$ & & $3.7(2.77-11.95)$ & \\
\hline Class IV & $0.75(0.14-3)$ & $<0.001$ & $8.44(3.72-26.5)$ & $<0.001$ \\
\hline Latent period (months) & $r=-0.489$ & $<0.001$ & $r=-0.228$ & 0.035 \\
\hline Manifest period (days) & $r=-0.974$ & $<0.001$ & $r=-0.478$ & $<0.001$ \\
\hline \multicolumn{5}{|l|}{ Schedule of surgery } \\
\hline Emergency $(n=50)$ & $0.6(0.1-3)$ & & $8.44(3.72-26.5)$ & \\
\hline Urgent $(n=45)$ & $0.17(0.03-1)$ & & $3.73(2.77-10.65)$ & \\
\hline Elective $(n=5)$ & $0.088(0.03-0.14)$ & $<0.001$ & $2.53(1.69-3.38)$ & $<0.001$ \\
\hline \multicolumn{5}{|l|}{ B-Operative and postoperative } \\
\hline Aortic cross clamp time (min) & $r=0.17$ & 0.12 & $r=0.204$ & 0.06 \\
\hline Cardiopulmonary bypass time (min) & $r=0.66$ & 0.013 & $r=0.35$ & 0.001 \\
\hline Mechanical ventilation & $r=0.31$ & 0.004 & $r=0.219$ & 0.043 \\
\hline Inotropes duration (h) & $r=0.23$ & 0.031 & $r=0.165$ & 0.128 \\
\hline ICU stay (days) & $r=0.18$ & 0.09 & $r=0.17$ & 0.12 \\
\hline Total hospital stay (days) & $r=0.035$ & 0.75 & $r=0.03$ & 0.79 \\
\hline Postoperative LVEF\% & $r=-0.252$ & 0.019 & $r=-0.424$ & $<0.001$ \\
\hline \multicolumn{5}{|l|}{ Mean gradient across prosthesis } \\
\hline Mitral & $r=0.33$ & $<0.006$ & $r=-0.308$ & 0.011 \\
\hline Aortic $(\mathrm{mm} \mathrm{Hg})^{*}$ & $r=-0.511$ & 0.075 & $r=-0.302$ & 0.316 \\
\hline \multicolumn{5}{|l|}{ Mortality } \\
\hline Mortalities $(n=18)$ & $0.75(0.1-3)$ & & $8.5(4.2-26.5)$ & \\
\hline Survivors $(n=68)$ & $0.35(0.03-2)$ & 0.002 & $6.27(1.69-13.84)$ & $<0.001$ \\
\hline \multicolumn{5}{|l|}{ Hospital survivors: } \\
\hline Complicated $(n=29)$ & $0.5(0.1-1.5)$ & & $6.89(2.86-13.84)$ & \\
\hline
\end{tabular}


Table 3 Index of deterioration versus EuroScore values (Continued)

\begin{tabular}{|c|c|c|c|c|}
\hline \multirow[b]{2}{*}{ Variable } & \multicolumn{2}{|c|}{ Index of deterioration (ID) } & \multicolumn{2}{|l|}{ EuroScore II } \\
\hline & Effect size & $P$ value & Effect size & $P$ value \\
\hline Uneventful cases $(n=39)$ & $0.2(0.03-2)$ & 0.011 & $4.4(1.69-13.84)$ & 0.006 \\
\hline
\end{tabular}

In our study, the median LPM that extended up to 30 years after native valve replacement was as early as 44.6 months, $85 \%$ of thrombosed prostheses were implanted in the mitral position, $60 \%$ of patients were females and two-thirds of cases had a positive history of noncompliance to OAC. Comparable results noting the timing of presentation (39+ 42 months), $85 \%$ female predominance, $85 \%$ mechanical prosthesis implanted in the mitral position, and $26 \%$ non-compliance to OAC were reported by Durrleman and colleagues [11]. Our 61.6\% rate of non-compliance is even higher than the $45 \%$ rate reported by Buttard and colleagues [13] but can be attributed to our series including 16 non-compliant pregnant patients. In fact, the ID was able to identify every one of those risk factors, being significantly higher in those patients who were older, of female gender, pregnant, having a history of non-compliance to OAC, or who developed PVT early after native valve replacement, compared to the other patients who were privileged by the absence of those risk factors. In concordance to others, the female gender $[8,11]$, early PVT after native valve replacement $[11,12]$, and non-compliance to OAC
$[11-13,17]$ were associated with worsening of outcomes and those were the factors that independently predicted a high ID in our study. In practice, a female presenting with PVT is expected to deteriorate 2 times faster than a male companion; this deterioration will unfortunately be doubled in case she was pregnant. Playing the same tune, a patient who is non-compliant to OAC is expected to deteriorate $50 \%$ faster than a compliant patient, with all other parameters being equal. As such, the ID may be useful to schedule patients for surgery and, in fact, the median ID of our emergency cases was 0.6, which was 3.5 times that of urgent cases and nearly 7 times that calculated in patients who were operated upon electively.

On the other hand, the ID correlated positively with major adverse operative and postoperative outcomes, such as the durations of cardiopulmonary bypass, mechanical ventilation, and need for inotropes. A high ID was an indicator of a limited improvement of LVEF\% after surgery, development of non-fatal complications, and mortality. The median ID of patients who developed non-fatal hospital complications was 2.5 times that

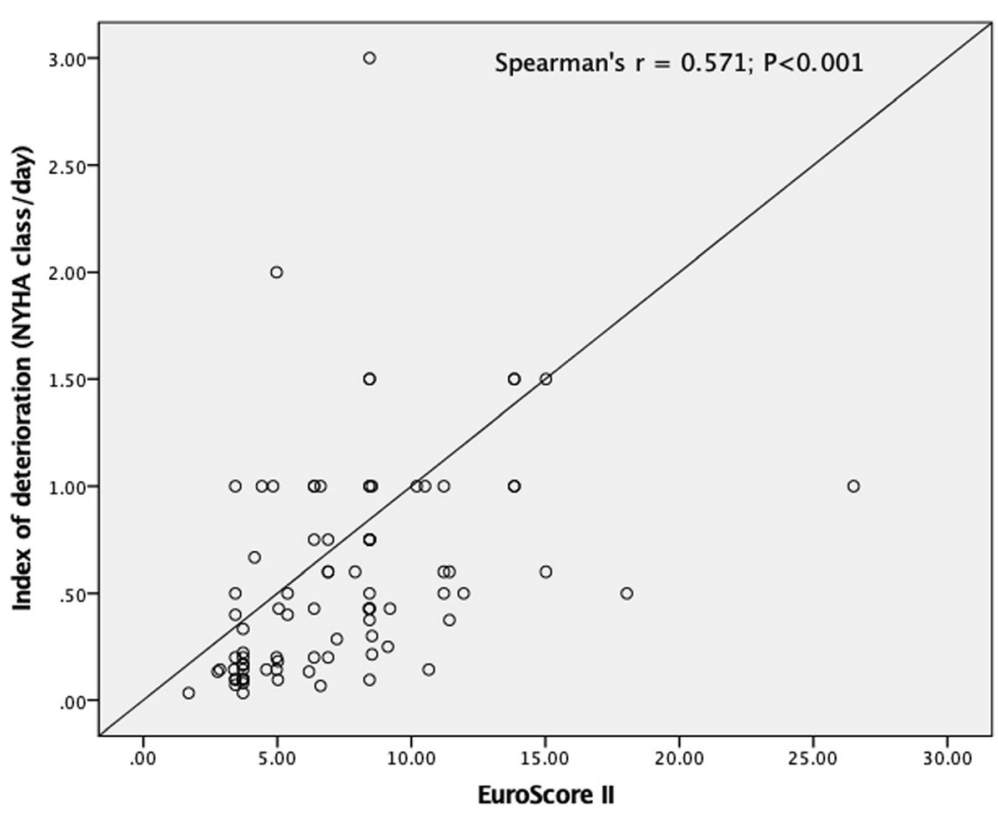

Fig. 2 Correlation between Index of deterioration and EuroScore II 
Table 4 Analysis of mortality

\begin{tabular}{|c|c|c|c|}
\hline Variable & Hospital survivors $(n=68)$ & Hospital mortalities $(n=18)$ & $P$ value \\
\hline \multicolumn{4}{|l|}{ A-Preoperative } \\
\hline Age (years) & $39(24-74)$ & $49.5(16-64)$ & 0.21 \\
\hline Female gender & $40(58.8 \%)$ & $11(61.1 \%)$ & 0.86 \\
\hline Pregnant patients & $12(17.6 \%)$ & $4(22.3 \%)$ & 0.9 \\
\hline \multicolumn{4}{|l|}{ Position of malfunctioning prosthesis } \\
\hline Mitral & $58(85.3 \%)$ & $15(83.3 \%)$ & \\
\hline Aortic & $10(14.7 \%)$ & $3(16.7 \%)$ & 0.86 \\
\hline NC-OAC $(n=47)$ & $40(50.6 \%)$ & $13(61.9 \%)$ & 0.36 \\
\hline Thromboembolic events & $8(11.8 \%)$ & $4(22.2 \%)$ & 0.26 \\
\hline \multicolumn{4}{|l|}{ NYHA class on admission } \\
\hline Class ॥ & $3(4.4 \%)$ & 0 & \\
\hline Class III & $34(50 \%)$ & $2(11.1 \%)$ & 0.004 \\
\hline Class IV & $31(45.6 \%)$ & $16(88.9 \%)$ & 0.003 \\
\hline Numerical NYHA class* & $3(2-4)$ & $4(2-4)$ & \\
\hline LPM (months) & $48(4-365)$ & $36(0.8-121.7)$ & 0.225 \\
\hline MPM (days) & $8.5(2-60)$ & $3.5(2-21)$ & 0.006 \\
\hline LVEF\% at presentation & $60(40-73)$ & $44.5(22-66)$ & 0.007 \\
\hline Mean pressure gradient across mitral prosthesis $(\mathrm{mm} \mathrm{Hg})^{* *}$ & $15(9-43)$ & $21(12-30)$ & 0.027 \\
\hline Mean pressure gradient across aortic prosthesis $(\mathrm{mm} \mathrm{Hg})^{* *}$ & $67(45-80)$ & $35(25-50)$ & 0.018 \\
\hline INR on admission & $1.5(0.7-3.4)$ & $1.8(1-4.4)$ & 0.078 \\
\hline Serum creatinine on admission (mg/dl) & $1.35(0.8-4.2)$ & $1.55(1-3.4)$ & 0.026 \\
\hline SGOT on admission (units/liter) & $40(15-120)$ & $72.5(24-150)$ & 0.002 \\
\hline \multicolumn{4}{|l|}{ Schedule of surgery } \\
\hline Emergency $(n=50)$ & $37(54.4 \%)$ & $14(77.8 \%)$ & \\
\hline Urgent $(n=45)$ & $29(42.6 \%)$ & $4(22.2 \%)$ & \\
\hline Elective $(n=5)$ & $2(2.9 \%)$ & 0 & 0.18 \\
\hline \multicolumn{4}{|l|}{ B-Operative and postoperative } \\
\hline \multicolumn{4}{|l|}{ Operative procedure } \\
\hline Replacement with a mechanical valve & $55(80.9 \%)$ & $14(77.8 \%)$ & \\
\hline Replacement with a tissue valve & $13(19.1 \%)$ & $3(16.7 \%)$ & \\
\hline Limited procedure without replacement & 0 & $1(5.6 \%)$ & 0.37 \\
\hline Aortic cross clamp time (min) & $60(37-140)$ & $87.5(50-200)$ & 0.02 \\
\hline Cardiopulmonary bypass time (min) & $102(55-205)$ & $160(100-360)$ & $<0.001$ \\
\hline Mechanical ventilation duration $(h)^{* *}$ & $24(8-120)$ & $33.5(6-168)$ & 0.54 \\
\hline Inotropes duration $(h)^{* * *}$ & $48(6-168)$ & $33.5(12-216)$ & 0.031 \\
\hline Postoperative LVEF\%*** & $55(40-70)$ & $37.5(15-45)$ & $<0.001$ \\
\hline ICU stay (days)*** & $4(1.5-14)$ & $2.5(2-39)$ & 0.025 \\
\hline Total hospital stay $($ days)*** & $10(6-21)$ & $3.5(1-40)$ & $<0.001$ \\
\hline
\end{tabular}

Values are presented as numbers (\%) or median (range)

$n$ number of patients, NC-OAC non-compliance to oral anticoagulants

*NYHA classes: II, III, and IV were assigned the numerical values 2, 3, and 4

**Excluding 5 patients with more than one prosthesis

${ }^{* * *}$ Calculated for the 68 operative survivors 


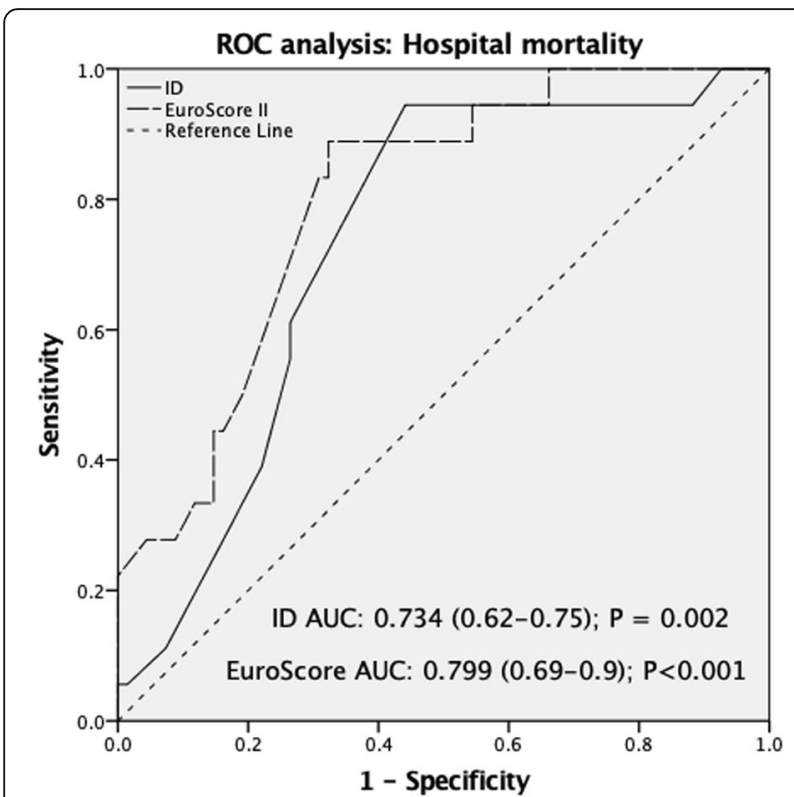

Fig. 3 ROC analysis: hospital mortality: index of deterioration versus Euroscore II

calculated for uneventful cases $(P=0.011)$ and the median ID of the 18 hospital mortalities was more than double that of the 68 survivors $(P=0.002)$. In practice, a patient, whose ID is 0.35 NYHA class/day or higher, is expected to develop non-fatal complications and mortality, with a sensitivity of $78.7 \%$ and specificity of $64.1 \%$. An ID $>0.46$ or higher is expected to unfortunately join mortality, with a sensitivity of $81 \%$ and specificity of

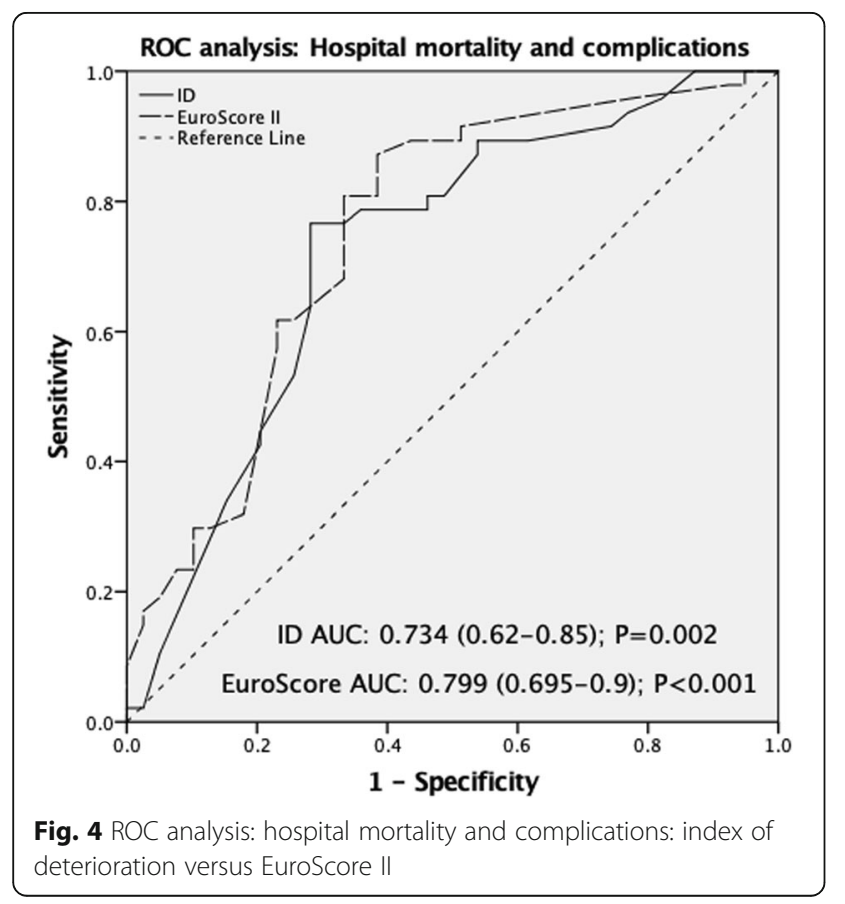

$62 \%$. We have previously tested the ID in a group of 75 patients presenting with different types of prosthetic malfunctions including PVT, prosthetic valve endocarditis, severe prosthetic dehiscence, and primary bioprosthetic failure. Due to the inclusion of a large number of patients with slowly progressive valve dehiscence, it was more suitable to calculate LPM in months rather than in days, giving larger crude ID values than the ones calculated in the actual study [18]. The ID calculated for different valve malfunctions also correlated positively and significantly with the early presentation of the malfunction, need for emergency, and longer durations of bypass and positive inotropes as well as hospital mortality [18].

Although the hospital mortality in our study was as heavy as $21 \%$, yet it was within the widely variable range reported to be as low as $5 \%$ in a small selected series of 20 patients [15] to $20.6 \%$ in a large series of 63 patients who developed mainly PVT due to pannus formation [14] to as much as $41 \%$ in a Canadian series of 39 patients [11]. Many risk factors have been identified to influence outcomes of surgery including early presentation $[8,11]$ myocardial depression, as indicated by low EF [14], and increased left ventricular diameter [14, 19], advanced NYHA class [20], prolonged cardiopulmonary bypass time [21], non-compliance to oral anticoagulation $(\mathrm{OAC})$ regimen $[11,13]$, and need for any additional procedure [20] Our study has shown that low LVEF\% on admission was the only independent predictor of hospital mortality and not the ID or the EuroScore II.

Although the STS risk score has been found to be highly effective for determining numerous outcomes such as short and long-term mortality, stroke, length of hospital stay and renal failure, prolonged ventilation, infection, and reoperation, yet it was modeled to fit specific surgical cases: isolated coronary artery bypass grafting (CABG), isolated aortic valve replacement (AVR), isolated mitral valve replacement (MVR), mitral valve repair (MV repair), CABG + AVR, CABG + MVR, and $C A B G+M V$ repair [22]. Because of this limitation, the STS risk scoring system cannot be used to study other types of cardiac surgery that are not yet included. On the other hand, the other most commonly used scoring system (the EuroScore II) that was designed to expect mortality in various surgical cases showed poor clinical relevance in the segment of patients where it is needed the most, i.e., the high risk and emergency cases [23-25]. Many studies have shown a significant discrepancy between observed and expected mortality in those patients when calculated by EuroScore II which sometimes overestimates [24] and in other times underestimates mortality [23] in high-risk patients [23, 24] as well as in emergency surgery [25]. In our study, the observed mortality was triple that expected by EuroScore II. Although it discriminated mortality and complications 
better than the ID, yet the wide gap between observed and expected values may be due to EuroScore II totally disregarding the important effect of time factor on outcome, whether the time delay between the implantation and the development of PVT $[8,10,11]$ : the LPM or the speed by which the patient deteriorates: the MPM; which is the basis upon which the ID was founded [18]. Another important forgotten factor is the patient's compliance to OAC $[11-15,17]$ which is an independent predictor of deterioration, as expressed in terms of ID.

\section{Conclusion}

The simple two-factors based ID (NYHA deterioration and time) was never thought as a replacement for the well-known multifactorial scoring systems, but as a useful numeric index to monitor deterioration and predicting hospital progression and outcomes in patients presenting with PVT. It raised questions about the need to include the time factor and non-compliance to OAC in predicting outcomes in those patients. However, larger studies are needed to show if the index of deterioration can be helpful to take decisions during the course of management of those patients.

\section{Abbreviations}

AUC: Area under the curve; AVR: Aortic valve replacement; CABG: Coronary artery bypass grafts; ID: Index of deterioration; LPM: Latent period of malfunction; LVEF \%: Left ventricular ejection fraction \%; MPM: Manifest period of malfunction; MVR: Mitral valve replacement; NYHA: New York Heart Association Classification; OAC: Oral anticoagulant; PVT: Prosthetic valve thrombosis; SJ: St Jude

\section{Acknowledgements}

Not applicable

\section{Authors' contributions}

All the authors shared in the study design, collecting data, and calculating the results. $A H, M G$, and $K R$ were the main persons in writing and revising this manuscript. AH was also responsible for the statistical analysis of the results. MG is the corresponding author; HM and KS were the surgeons operating on the included patients. All the authors have read and approved the submitted version of the manuscript

\section{Funding}

Not applicable

\section{Availability of data and materials}

Available for public share in the main manuscript and the tables and figures.

\section{Ethics approval and consent to participate}

This study was approved by the ethical committee of the Faculty of Medicine, Ain-Shams University (Ref FMASU MD 185/2017).

Written informed consent was obtained from all participants in this study.

\section{Consent for publication}

Not applicable

\section{Competing interests}

The authors declare that they have no competing interests.
Received: 3 August 2020 Accepted: 14 September 2020

Published online: 29 September 2020

\section{References}

1. Yau TM, El-Ghoneimi YA, Armstrong S, Ivanov J, David TE (2000) Mitral valve repair and replacement for rheumatic disease. J Thorac Cardiovasc Surg. 119:53-60

2. Templeton GF. The two-steps approach for transforming continuous variables to normal: implications and recommendations for IS research. Communications of the association for information systems: vol 28, Article 4: DOI: https://doi.org/10.17705/1CAIS.02804. Available at: https://aisel.aisnet. org/cais/vol28/iss $1 / 4$

3. Laplace G, Lafitte S, Labèque JN et al (2004) Clinical significance of early thrombosis after prosthetic mitral valve replacement: a postoperative mono- centric study of 680 patients. J Am Coll Cardiol 43:1283-1290

4. Wolberg AS, Aleman MM, Leiderman K et al (2012) Procoagulant activity in hemostasis and thrombosis: Virchow's triad revisited. Anesth Analg 114:275-285

5. Roudaut R, Serri K, Lafitte S (2007) Thrombosis of prosthetic heart valves: diagnosis and therapeutic considerations. Heart 93:137-142

6. Zoghbi WA, Chambers JB, Dumesnil JG et al (2009) Recommendations for evaluation of prosthetic valves with echocardiography and Doppler ultrasound. J Am Soc Echocardiogr 22:975-1014

7. Sun JC, Davidson MJ, Lamy A et al (2009) Antithrombotic management of patients with prosthetic heart valves: current evidence and future trends. Lancet 374:565-576

8. Blackstone EH, Kirklin JW (1985) Death and other time - related events after valve replacement. Circulation 72:753-767

9. Husebey DG, Pluth JR, Pielher JM, Schaff HV, Orzulak TA,Puga FY et al. Reoperation on prosthetic heart valves: an analysis of risk factors in 552 patients. JThorac Cardivasc Surg 1983; 86:543-552.

10. Lin SS, Tiong IY, Asher CR et al (2000) Prediction of thrombus-related mechanical prosthetic valve dysfunction using transesophageal echocardiog- raphy. J Am Coll Cardiol 86:1097-1101

11. Nicolas Dürrleman, Michel Pellerin, Denis Bouchard, Yves Hébert, Raymond Cartier, Louis P Perrault, Arsène Basmadjian, Michel Carrier. Prosthetic valve thrombosis: twenty-year experience at the Montreal Heart institute, J Thoracic Cardiovascular Surgery 2004; 127, 5: 1388-1392.

12. Lin SS, Tiong IY, Asher CR et al (2000) Prediction of thrombus-related mechanical prosthetic valve dysfunction using transesophageal echocardiog- raphy. J Am Coll Cardiol 86:1097-1101

13. Buttard P, Bonnefoy E, Chevalier P, Marcaz PB, Robin J, Obadia JF et al (1997) Mechanical cardiac valve thrombosis in patients in critical hemodynamic compromise. Eur J Cardiothorac Surg. 11:710-713

14. Toker ME, Eren E, Balkanay M, Kirali K, Yanartas M, Çaliskan A et al (2006) Multivariate analysis for operative mortality in obstructive prosthetic valve dysfunction due to pannus and thrombus formation. Int Heart J 47:237-245

15. Ahn H, Kim KH, Kim KC, Kim CY (2008) Surgical management of mechanical valve thrombosis: twenty-six years' experience. J Korean Med Sci 23:378-382

16. Gohlke-Bärwolf C (2000) Anticoagulation in valvar heart disease: new aspects and management during non-cardiac surgery. Heart 84:567-572

17. Sahnoun-Trabelsi I, Jimenez M, Choussat A et al (2004) Thromboses de prothèses valvulaires cardiaques chez la femme enceinte. Archives des maladies du coeur et des vaisseaux. 97:305-310

18. A. Hassouna. Index of deterioration of patients with prosthetic valve malfunctions. The Bulletin of the Egyptian Society of Cardiothoracic Surgery 1995; 3: 75 - 87.

19. Bortolotti U, Milano A, Mossuto E, Mazzaro E, Thiene G, Casarotto D (1994) Early and late outcome after reoperation for prosthetic valve dysfunction: analysis of 549 patients during a 26-year period. J Heart Valve Dis 3:81-87

20. Muhammad Sharoz Rabbani, Iffan Qadir, Yasir Ahmed, Marrium Gul, Hasanat Sharif Heart valve surgery: EuroSCORE vs. EuroSCORE II vs. Society of Thoracic Surgeons score Heart International 2014; 9(2):53-58.

21. Carlos Manuel de Almeida Brandão, Pablo Maria Alberto Pomerantzeff, Luciano Rapold Souza, Flavio Tarasoutchi, Max Grimberg, Jose' Antonio Franchini Ramires, Se'rgio Almeida de Oliveira. Multivariate analysis of risk factors for hospital mortality in valvular reoperations for prosthetic valve dysfunction. Euro J Cardio-thoracic Surgery 2002; 22: 922-926.

22. Online STS risk calculator. http://riskcalc.sts.org/stswebriskcalc/. Accessed 10 June 2020. 
23. Ranucci M, Di Dedda U, Castelvecchio S et al (2016) In search of the ideal risk-scoring system for very high-risk cardiac surgical patients: a two-stage approach. J Cardiothorac Surg 11:13

24. Casalino S, Stelian E, Mangia F, Novelli E, Renzi L, Alessi C, Diena M (2007) Evaluation of the EuroSCORE in high risk patients undergoing cardiac surgery. Euro J Anaesthesiol 24:20

25. Grant SW, Hickey GL, Dimarakis L, Cooper G, Jenkins DP, Uppal R, Buchan L, Bridgewater B (2013) Performance of the EuroSCORE models in emergency cardiac surgery. Circulation 6(2):178-185

\section{Publisher's Note}

Springer Nature remains neutral with regard to jurisdictional claims in published maps and institutional affiliations.

\section{Submit your manuscript to a SpringerOpen ${ }^{\circ}$ journal and benefit from:}

- Convenient online submission

Rigorous peer review

- Open access: articles freely available online

High visibility within the field

- Retaining the copyright to your article

Submit your next manuscript at $\boldsymbol{\wedge}$ springeropen.com 